\title{
Index rerum ad Vol. 153
}

\section{Confecit C. Loeb-Schürch}

$(\mathrm{B}) \approx$ Buchbesprechungen - Livres nouveaux - Book reviews $(\mathrm{V})=$ Vortrag - Communication Report

Abflußwege des Kammerwassers,

v. Drainage Ablatio retinae, v. Lichtkoagulation,

Retinal detachment surgery Abt-Letterer-Siwe disease; chorio-

retinal degeneration of the pigmentary type in the Abt-Letterer-

Siwe disease, 241 Accidents vaccinaux; deux points à

souligner en matière d'ophtalmo-

accidents vaccinaux, 198 Accommodation, v. Amblyopy Acides amines, v. Linsenalterung

Acuité visuelle, v. Functions visuellc·s,

Partially sighted children, Visual

acuity Adaptation, v. Amblyopy Aderhaut, v. Chorioidal detachment,

Pressure pulse Affe, v. Monkey vitreous Ageing of lens, v. Linsenalterung Albuminoid, v.

Linsenalterung Allergie, v. Autoallergic ocular

diseases Amblyopy, v. Partially sighted

children, Retinal correspondence Amblyopy; the problem of the reversing phenomenon' in

amblyopy, 73 Aminosäuren, v. Linsenalterung Anesthesia, retrobulbar, v. Retro-

bulbar hemorrhage Anésthésie, v. Fluothane, Neuroleptoanalgesia Anomalous retinal

correspondence

and monolateral squint, 179 Anterior chamber, v. Invasion

épithéliale, Osmothérapie Anterior segment; changes on the

anterior segment of the eye after

retinal detachment surgery, 164 Anti-microbial properties of monkey

vitreous, 100 Applanation tonometer; new ap-

planation tonometer for operating room, 225

Aqueous humour, v. Drainage,

Guanethidine, Invasion épithéliale, Osmothérapie

Athalamie postopératoire, v. Osmothérapie

Atrophie optique, v. Abt-Letterei Siwe disease, Quinine

Alropin, v. Fluothane

Augenbinnendruck, v. Intraocular-druck

Augenblase; die Entwicklungs-bewegungen der menschlichen Augenblase. Hire Bedeutung für die í, 'ühe Gesichtsbildung, 291

Augenhintergrundsveränderungen, v. Abt-Letterer-Siwe disease, Fluoreszenz-Photographie, Licht-koagulatkn, Neuritis nervi optici, Papillenoedem, Quinine, Retinitis centralis serosa Augenkomplikationen, v. Accidents vaccinaux, Chorioidal detachment, Invasion épithéliale, Lichtkoagulation, Lyell-Syndrom, Quinine, Osmothérapie, Retinal detachment, Retrobulbar hemorrhage 
Augenlänge, v. Coefficient de rigidité

Augenmuskeln, v. Motilitätsschema, Muskeldystrophie

Autoallergic ocular diseases; clinical problems of autoallergic ocular diseases, 93

Barbiturates, v. Neuroleptoanalgesia

Base du crane, v. Fractures

Binocular relationship; a test for binocular relationship, 197

Biochemie, v. Cataract, Guane-thidine, Hexose penetration, Linsenalterung, Sodium iodate,

Sulfur

476

Index rerum

ad Vol. 153

Biomicroscopie, v. Embryotoxon Biomicroscopie; Manuel de biomicroscopie oculaire, 79 (B)

Blood plasma dialyzate, v. Cataract Blutungen in den Glaskörper; zur

Therapie der Blutungen in den

Glaskörper, 107 Blutung, retrobulbäre, v. Retrobulbar

hemorrhage Book reviews, 79 (B), 239 (B),

400 (B), 474 (B) Bruch'sche Membran, v. Retinitis

centralis serosa Buchbesprechungen, 79 (B), 239 (B),

400 (B), 474 (B)

Candida albicans; die experimentelle endogene Entzündung des Augen-innern durch Candida albicans, 400 (B)

Carcinoma; muco-epidermoid carcinoma of the lacrimal gland, 184

Cataract; on the ability of the blood plasma dialyzate of patients with senile cataract to influence

'in vitro' the activity of some de-hydrogenases, 47

Cataract, v. LinsenaUening

Central retinal artery; occlusion of the central retinal artery following retrobulbar hemorrhage,

191

Central serous retinopathy, v. Retinitis centralis serosa

Chambre antérieure, v. Invasion épithéliale, Osmothérapie

Champ de fixation, v. Field of fixation

Champ visuel, v. Field of fixation, Neuritis nervi optici, Quinine

Chinin, v. Quinine

Chirurgie oculaire, v. Applanation tonometer, Chorioidal detachment, Fluothane,

Neuroleptoanalgesia, Osmothérapie, Retinal detachment surgery

Chirurgische Eingriffe am Auge; zur Frage der Fluothane-Narikose für chirurgische Eingriffe am

Auge, 200

Chlorpromazine; influence of Chlor-promazine intracisternal injection

on the intraocular pressure of the rabbit, 393

Chorioidea, v. Chorioidal detachment, Pressure pulse

Chorioidal detachment after irid-encleisis; its occurrence and the results of conservative

treatment, 6

Chronisch progressive Muskel-dystrophie; elektronenmikrosko-pische Befunde bei 3 Fallen von chronisch progressiver oculärer Muskeldystrophie, 439

Circulation de la papille, v. Papillen-kreislauf

Coagulation à la lumière, v. Licht-koagulation, Retinitis centralis serosa 
Coefficient de rigidité; relation entre le coefficient de rigidité et la longueur de Гæil mesurée par Techographie ultrasonique, 355

Collagenosis, v. Wegenersche Granulomatose

Complications oculaires, v. Accidents vaccinaux, Chorioidal detachment, Invasion épithéliale, Lichtkoagula-tion, Lyell-Syndrom, Osmothérapie, Quinine, Retinal detachment surgery,

Retrobulbar hemorrhage

Computer, v. Papillenkreislauf

Congenital neuro-lacrimal syndrome Paroxysmal lacrimation while eating with external rectus palsy, 174

Congrès, v. Ophthalmological Society of the United Kingdom

Cornea, v. Coefficient de rigidité, Dystrophia cornealis, Embryotoxon, Hornhaut-Interferometer

Corneal graft, v. Autoallergic ocular diseases

Corpus vitreum, v. Glaskörperblutung, Monkey vitreous, Papil-lenoedem

Correspondance rétinienne, v. Retinal correspondence

Corticosteroide, v. Glaskörperblutung

Corticosteroidtherapie; intravenose Corticosteroidtherapie bei Neuritis nervi optici und Uveitis, 15

Cristallin, v. Hexose penetration, Linsenalterung, Spherophakia

Index rerum ad Vol. 153

477

Décollement de la chorioïde,

v. Chorioidal detachment Décollement rétinien, v. Licht-

koagulation, Retinal detachment

surgery Degénérescence maculaíre, v. Retinitis

centralis serosa Degénérescence rétinienne, v. Abt-

Letterer-Siwe disease, Sodium

iodate Dehydrogenases, v. Cataract Diagnostik, v. Autoallergic ocular

diseases, Fluoreszenzphotographie,

Mass screening, Motilitätsschema Dialyzate of blood plasma,

v. Cataract Disc oedema, v. Papillenkreislauf,

Papillenoedem Drainage of aqueous humour;

concerning the posterior routes

for the drainage of aqueous

humour, 215 Dystrophia cornealis filiformis pro-

funda. Hornhautdystrophie von

Maeder und Danis, 321 Dystrophie musculaire, v. Muskel-

dystrophie

Echographie ultrasonique, v. Coefficient de rigidité Ecoulement de Thumeur aqueuse,

v. Drainage Eiweiß, v. Linsenalterung Elektromyogramm, v. Muskel-

dystrophie Elektronenmikroskopie, v. Muskel-

dystrophie, Papillenoedem Electro-oculogramm, v. Quinine Elektroretinogramm, v. Abt-

Letterer-

Siwe disease, Optical aids, Quinine EMG, v. Muskeldystrophie Embryologie, v. Augenblase

Embryotoxon; le tableau bio-

microscopique de Гembryotoxon 
antérieur, 1 Encyclopedia; the encyclopedia of physics, 240 (B) EOG, v. Quinine

Epidemiologie, v. Optic neuritis Epithelinvasion, v. Invasion épithé-

liale

Epithelioma; benign epithelioma of the iris, 286

Epithelium pigmentaire, v. Retinitis centralis serosa, Sodium iodate

ERG, v. Abt-Letterer-Siwe disease, Optical aids, Quinine

Exophthalmos, v. Retrobulbar hemorrhage, Wegenersche Gra-nulomatose

Exophthalmos; unilateral pulsating exophthalmos in von Reckling-hausen's disease, 409

Experimentelle Forschung, v. Chlor-promazine, Guanethidine, Hexose penetration,

Linsenalterung, Monkey vitreous, Papillenkreislauf, Plasmadialysat, Sodium iodate, Sulfur

Fehlerquellen der Fluoreszenz-Fotografie, 349

Field of fixation measured by the Goldmann perimeter. Practical measurements of field of

fixation by the application of the Goldmann perimeter, 460

Fixation, eccentric, v. Binocular relationship

Fixationsfeld, v. Field of fixation

Fluorescein, v. Fluoreszenz-Foto-grafie, Retinitis centralis serosa

Fluoreszenz-Fotografie; Fehlerquellen der Fluoreszenz-Fotografie, 349

Fluoreszenzphotographie, v. Retinitis centralis serosa

Fluothane; zur Frage der Fluothane-Narkose für chirurgische Eingriffe am Auge, 200

Fonctions musculaires, v. Motilitätsschema, Muskeldystrophie

Fonctions visuelles; etudes des fonctions visuelles dans deux cas d'intoxication par la quinine, 324

Fortbildung und Orientierung, 309, 467

Foveola, v. Visual acuity

Foveal after-images, v. Retinal correspondence

Fractures of the base of the skull, 400 (B)

Ophthalmologica, Vol. 153, No. 6 (1967)

38

478

Index rerum

ad Vol. 153

Fundus, v. Abt-Letterer-Siwe disease, Fluoreszenz-Photographie, Licht-koagulation, Neuritis nervi optici, Papillenoedem, Quinine, Retinitis centralis serosa

Further studies and other information, 309, 467

Galactose, v. Hexose penetration Gefäße, v. Embryotoxon, Papillen-

kreislauf, Papillenoedem, Pressure

pulse Gesichtsfeld, v. Field of fixation,

Neuritis nervi optici, Quinine Glande lacrymale, v. Lacrimal gland Glaskörper, v.

Glaskörperblutung,

Monkey vitreous, Papillenoedem Glaskörperblutung; zur Therapie der

Blutungen in den Glaskörper, 107 Glaucoma, v. Mass screening Glaucome secondaire, v. Osmothérapie Glucose, v. Hexose penetration Glycerin, v. Osmothérapie Glycerol, v. Osmothérapie

Goldmann perimeter, v. Field of 
fixation Granulomatosis, v. Wegenersche

Granulomatose Greffe cornéenne, v. Autoallergic

ocular diseases Guanethidine; the effects of local

administration of Guanethidine

and BW $467 \mathrm{C} 60$ on the aqueous

humour composition in rabbit, 385

Hémorrhagie rétrobulbaire, v. Retro-bulbar hemorrhage

Hérédité, v. Dystrophia cornealis filiformis profunda

Hexose penetration; a method for studying hexose penetration into the lens using the sorbitol

pathway as a trapping mechanism, 423

Hilfsmittel, optische, v. Optical aids

Histologie, v. Augenblase,

Carcinoma, Epithelioma, Invasion épithéliale, Lyell-Syndrom, Muskeldystrophie, Von Reckling-

hausen's disease

Hornhaut, v. Coefficient de rigidité, Dystrophia cornealis, Embryotoxon, Hornhaut-

Interferomeler

Hornhaut-Interferometer; Gedanken über den Bau eines Hornhaut-Interferometers, 43

Hornhauttransplantat, v. Autoallergic ocular diseases

Humeur aqueuse, v. Drainage,

Guanethidine, Invasion épithéliale, Osmothérapie

Hypermetropie, v. Coefficient de rigidité

Hypotonia oculi, v. Neurolepto-analgesia

Impfung, v. Accidents vaccinaux

Injection, v. Chlorpromazine, Corti-costeroidtherapie

In memoriam Professor Dekking, 161

Instrument, v. Applanationtono-meter

Interferometer, v. Hornhaut-Interferometer

Intoxication, v. Quinine

Intracisternal injection, v. Chlorpromazine

Intraoculardruck, v. Applanation tonometer, Chlorpromazine, Fluothane, Guanethidine, Invasion épithéliale, Mass screening, Pressure pulse

Intraocular pressure, v. Ocular tension

Invasion épithéliale de la chambre antérieure: confirmation anato-mique par Гexamen

cytologique de Thumeur aqueuse, 467

Iridenkleisis, v. Chorioidal detachment

Iris, v. Drainage, Epithelioma

J181, v. Sodium iodate Jodat, v. Sodium iodate

Kammerwasser, v. Drainage,

Guanethidine, Invasion épithéliale,

Osmothérapie Kaninchen, v. Chlorpromazine,

Guanethidine, Sodium iodate Katarakt, v. Cataract Katarakt, v. Linsenalterung Kollagenose, v.

Wegenersche

Granulomatose

Index rerun! ad Vol. 153

479 
Kongreß, v. Ophthalmological Society of the United Kingdom

Korrespondenz, retinale, v. Retinal correspondence

Krebs, v. Carcinoma

Lacrimal gland; muco-epidermoid carcinoma of the lacrimal gland, 184

Lacrimation, v. NeurO-lacrimal syndrome

Länge des Auges, v. Coefficient de rigidité

Lapin, v. Chlorpromazine, Guane-thidine, Sodium iodate

Length of the eye, v. Coefficient de rigidité

Lens, v. Hexose penetration, Linsen-alterung, Spherophakia

Libri, 79 (B), 239 (B),400 (B)

Lichtbedarf, v. Light requirement

Lichtkoagulation; über Spätkompli-kationen nach prophylaktischer Lichtkoagulation der

Netzhaut, 401

Lichtkoagulation, v. Retinitis centralis serosa

Light requirement; an investigation into the usefulness of optical aids and the light requirements in a school for partially sighted children in the Netherlands, 129

Linse, v. Hexose penetration, Linsen-alterung, Spherophakia

Linsenalterung; Untersuchungen der Aminosäurenzusammensetzung klarer und kataraktöser

mensch-licher Linsen und ihre Bedeutung für das Verständnis der Eiweiß-transformation

während der Linsenalterung, 141

Livres nouveaux, 79 (B), 239 (B), 400 (B), 474 (B)

Longueur de Гæil, v. Coefficient de

rigidité Lumière, besoin de, v. Light requirement

Lyell-Syndrom; Augenveränderungen beim Lyell-Syndrom, 81

Maculadegeneration, v. Retinitis centralis serosa

Macula lutea, v. Visual acuity

Maeder und Danis; dystrophia cornealis filiformis profunda. Hornhautdystrophie von Maeder und Danis, 321

Malformations oculaires, v. Spherophakia

Mass screening of adults for glaucoma, 336

Membrane de Bruch, v. Retinitis centralis serosa

Mesenchym, v. Augenblase

Mic Гoscopie électronique, v. Muskel-dystrophie, Papillenoedem

Mißbildungen, v. Spherophakia

Monkey vitreous; anti-microbial properties of monkey vitreous, 100

Monolateral squint; anomalous retinal correspondence and mono-lateral squint, 179

Motilitätsschema; über ein neues dynamisches Motilitätsschema, 37

Moyens optiques, v. Optical aids

Muco-epidermoid carcinoma of the lacrimal gland, 184

Muscular scheme, v. Motilitätsschema

Muskeldystrophie; elektronen-mikroskopische Befunde bei 3 Fallen von chronisch progressiver oculärer Muskeldystrophie, 439

Muskelfunktionen, v. Motilitätsschema, Muskeldystrophie

Myopie, v. Coefficient de rigidité

Nachbild, foveales, v. Retinal correspondence Narkose, v. Fluothane, Neurolepto-

analgesia Natrium-Jodat, v. Sodium iodate Netzhaut, v. Lichtkoagulation, 
Quinine Neuritis nervi optici, v. Cortico-

steroidtherapie, Optic neuritis Neuritis retrobulbaris, v. Optic

neuritis Neurofibromatosis, v. Von Reckling-

hausen's disease Neuro-lacrimal syndrome; congenital

neuro-lacrimal syndrome.

Paroxysmal lacrimation while

eating with external rectus palsy,

174

480

Index rerum ad Vol. 153

Neuroleptoanalgesia; the use of Neu Ooleptoanalgesia in ophthalmic surgery, 431

Névrite rétrobulbaire, v. Optic neuritis

Occlusion of the central retinal artery following retrobulbar hemorrhage, 191

Ocular tension, v. Applanation tonometer, Chlorpromazine, Fluothane, Guanethidine, Invasion

épithéliale, Mass screening, Pressure pulse

Oculo-cardialer Reflex, v. Fluothane

Oedème papillaire, v. Papillenoedem

Operation, v. Applanation tonometer, Chorioidal detachment, Fluothane, Neuroleptoanalgesia,

Osmo-therapie, Retinal detachment surgery

Ophthalmie sympathique, v. Auto-allergic ocular diseases

Ophthalmological Society of the United Kingdom, 86th annual congress (1966), 78

Ophthalmoplegia externa progressiva Graefe, v. Muskeldystrophie

Optical aids; an investigation into the usefulness of optical aids and the light requirements in a school for partially sighted children in the Netherlands, 129

Optic neuritis; epidemiological survey of optic neuritis in Italy in the years from 1958 to 1962 , 51

Optic vesicle, v. Augenblase

Opticusatrophie, v. Abt-Letterer-Siwe disease, Quinine

Osmothérapie; rétablissement de la chambre antérieure par Гosmo-thérapie (glycérol), 277

Palsy, v. Motilitätsschema, Muskeldystrophie, v. Neuro-lacrimal syndrome

Papille, v. Papillenkreislauf, Papillenoedem

Papillenkreislauf; Betrachtungen zum Systemprogramm «Papillen-kreislauf», 112

Papillenoedem; elektronenmikrosko-pische Betrachtungen an der

Papille des Rattenauges und beim Papillenoedem des Menschen, 367

Papillitis, v. Optic neuritis

Parese, v. Motilitätsschema, Muskeldystrophie, Neuro-lacrimal syndrome

Partially sighted children; an investigation into the usefulness of optical aids and the light

requirements in a school for partially sighted children in the Netherlands, 129

Perfectionnement et orientation, 309, 467

Perimetrie, v. Field of fixation, Neuritis nervi optici, Quinine

Photocoagulation, v. Lichtkoagula-tion, Retinitis centralis serosa

Photographie, v. Fluoreszenz-Fotografie

Physics; the encyclopedia of physics, 240 (B)

Physiology of the eye. Clinical application, 80 (B)

Pigmentdegeneration der Netzhaut, v. Abt-Letterer-Siwe disease, Sodium iodate 
Pigment epithelium, v. Sodium iodate

Plasmadialyzate; on the ability of the blood plasma dialyzate of patients with senile cataract to influence 'in vitro' the activity of some dehydrogenases, 47

Pleoptik und Orthoptik, 474 (B)

Post-image fovéenne, v. Retinal correspondence

Pressure pulse; on the origin of ocular pressure pulse, 29

Proceedings, v. Ophthalmological Society of the United Kingdom

Professor Dekking; in memoriam Professor Dekking, 161

Progressive external ophthalmoplegia, v. Muskeldystrophie

Protein, v. Linsenalterung

Pulsdruck, ocularer, v. Pressure pulse

Quinine; etudes des functions visuelles dans deux cas d'intoxi-cation par la quinine, 324

Index rerum

ad Vol. 153

481

Rabbit, v. Chlorpromazine, Guane-thidine, Sodium iodate

Radioaktives Na-Jodat, v. Sodium iodate

Rat, v. Hexose penetration, Papillen-oedem

Renversement des crochets de Pflüger, v. Amblyopy

Reticuloendotheliose, v. Abt-Letterer-Siwe disease

Retina, v. Lichtkoagulation, Quinine

Retinal correspondence; anomalous retinal correspondence and mono-lateral squint, 179

Retinal degeneration, v. Abt-Letterer-Siwe disease, Sodium iodate

Retinal detachment, v. Lichtkoagulation, Retinal detachment surgery

Retinal detachment surgery; changes on the anterior segment of the eye after retinal detachment surgery, 164

Retinitis centralis serosa; zu $\gamma$ Patho-genese und Therapie der soge-nannten Retinitis centralis serosa, 259

Reversing phenomenon; the problem of the 'reversing phenomenon' in amblyopy, 73

Recherches expérimentales, v. Chlorpromazine, Guanethidine, Hexose penetration, Linsenalterung, Monkey vitreous, Papillenkreislauf, Plasmadialyzate, Sodium iodate, Sulfur

Retrobulbäranaesthesie, v. Retrobulbar hemorrhage

Retrobulbar hemorrhage; occlusion of the central retinal artery following retrobulbar hemorrhage, 191

Retrobulbärneuritis, v. Optic neuritis

Rigiditätskoeffizient, v. Coefficient de rigidité

Schädelbasisfrakturen, 400 (B) Schema de motilité oculaire, 37 Schielen, v. Amblyopy,

Monolateral

squint, Strabismes Schwefel, v. Sulfur Sehfunktionen, v. Fonctions visuelles

Sehschärfe, v. Fonctions visuelles, Partially sighted children, Visual acuity

Sehschwache, v. Partially sighted children

Sekundärglaukom, v. Osmothérapie

Singe, v. Monkey vitreous

Sodium iodate; the penetration of J131 labeled sodium iodate into the ocular tissues and fluids, 229 
Sorbitol, v. Hexose penetration

Soufre, v. Sulfur

Spherophakia, 419

Sphingolipidosis, v. Abt-Letterer-Siwe disease

Sphygmographie, v. Pressure pulse

Squint, v. Amblyopy Monolateral squint, Strabismes

Staphylococcus pyogenes, v. Monkey vitreous

Stauungspapille, v. Papillenkreislauf

Strabismes. Hétérophories, paralysies oculo-motrices, 239 (B)

Strabismus, v. Amblyopy, Mono-lateral squint, Strabismes

Sulfur content in the normal human eye, 158

Surgery, v. Applanation tonometer, Chorioidal detachment, Fluothane, Neuroleptoanalgesia,

Osmothérapie, Retinal detachment surgery

Sympathetic ophthalmia, v. Auto-allergic ocular diseases

Syndrome, v. Lyell-Syndrom, Neuro-lacrimal syndrome

Systemprogramm; Betrachtungen zum Systemprogramm «Papillenkreislauf», 112

Tapeto-retinal degeneration, v. Abt-Letterer-Siwe disease

Tension oculaire, v. Applanation tonometer, Chlorpromazine, Fluothane, Guanethidine, Invasion épithéliale, Mass screening, Pressure pulse, Retrobulbar hemorrhage

Test for binocular relationship, 197

Therapie, v. Chorioidal detachment, Corticosteroidtherapie, Glaskörper-blutung, Osmothérapie, Retinitis centralis serosa

482

Index rerum ad Vol. 153

Thesaurismosis, v. Abt-Letterer-Siwe

disease Thorotrast, v. Drainage Thrombose de la veine rétinienne

centrale, v. Papillenkreislauf Tonometer, v. Applanation tonometer Toxic epidermal necrolysis, v. Lyell-

Syndrom Tränen, v. Neuro-lacrimal syndrome Tränendrüse, v. Lacrimal gland Tumor, v.

Carcinoma, Epithelioma Tumoren des Gesichtsschädels, die,

$474(\mathrm{~B})$

Ultraschall-Echographie, v. Coefficient de rigidité

Ultrasonographie, v. Coefficient de rigidité

Umkehrphänomen Pflüger'scher Haken, v. Amblyopy

Untersuchungstechnik, v. Binocular relationship, Fluoreszenz-Photo-graphie, Hornhaut-

Interferometer, Mass screening, Pressure pulse, Visual acuity

Uveitis, v. Corticosteroidtherapie, Autoallergic ocular diseases

Vaccination, v. Accidents vaccinaux

Vaisseaux, v. Embryotoxon, Papillenkreislauf, Papillenoedem, Pressure pulse

Varia, 80

Vererbung, v. Dystrophia cornealis filiformis profunda

Vergiftung, v. Quinine

Verhandlungsberichte, v. Ophthal-mological Society of the United Kingdom

Vésicule optique, v. Augenblase

Vessels, v. Embryotoxon, Papillenkreislauf, Papillenoedem, Pressure pulse 
Vieillissement du cristallin, v. Lin-senalterung

Visual acuity; central and peripheral visual acuity, 66

Visual acuity, v. Fonctions visuelles, Partially sighted children

Visual field, v. Field of fixation, Neuritis nervi optici, Quinine

Visual functions, v. Fonctions visuelles

Vitreous body, v. Glaskörperblutung, Monkey vitreous, Papillenoedem

Vogt; Alfred Vogt in Basel, 79 (B)

Volume oculaire, v. Coefficient de rigidité

von Recklinghausen's disease; unilateral pulsating exophthalmos in von Recklinghausen's

disease, 409

Vorderkammer, v. Invasion épithé-liale, Osmothérapie

Wegenersche Granulomatose; Augen-symptome der Wegenerschen Granulomatose. Bericht über einen Fall, 309

Z. Zentralarterienverschluß, v. Occlusion of the central retinal artery

Zentralvenen-Thrombose, v. Papillenkreislauf 